Data Article

\title{
Evaluation of the performance of two tuberculosis interferon gamma release assays (IGRA-ELISA and T-SPOT.TB) for diagnosing Mycobacterium tuberculosis infection
}

\author{
Linchuan Wang ${ }^{\mathrm{a}}$, Xu-dong Tian ${ }^{\mathrm{a}, 1}$, Yan Yu ${ }^{\mathrm{b}, *}$, Wei Chen ${ }^{\mathrm{a}, *}$ \\ ${ }^{a}$ The First Affiliated Hospital of Xi'an Jiaotong University, Xi'an, Shaanxi Province, China \\ ${ }^{\mathrm{b}}$ Honghui Hospital, Xi'an Jiaotong University, Xi'an, Shaanxi Province, China
}

\section{A R T I C L E I N F O}

\section{Article history:}

Received 11 January 2018

Received in revised form

16 March 2018

Accepted 24 August 2018

Available online 31 August 2018

Keywords:

Mycobacterium tuberculosis

IGRA-ELISA

T-SPOT.TB

\begin{abstract}
A B S T R A C T
This data contains information from 3727 patients and shows the performance of two IGRAs tests (T-SPOT.TB and IGRA-ELISA) used in China for screening and diagnostic Mycobacterium tuberculosis infection. The positive results were divided into four groups according with the test values, and the proportions of positives in each group were compared. The positive predictive values (PPVs) at different cutoffs for diagnostic active TB and value change trend for the two IGRAs tests were analyzed.

(c) 2018 The Authors. Published by Elsevier Inc. This is an open access article under the CC BY license (http://creativecommons.org/licenses/by/4.0/).
\end{abstract}

\section{Specifications Table}

$\begin{array}{ll}\text { Subject area } & \text { Biology } \\ \text { More specific subject area } & \text { Microbiology } \\ \text { Type of data } & \text { Table and figure } \\ \text { How data was acquired } & \text { LIS and HIS of the hospital } \\ \text { Data format } & \text { Raw and analyzed data }\end{array}$

DOI of original article: https://doi.org/10.1016/j.cca.2018.01.014

* Corresponding authors.

E-mail addresses: yu.yan74@163.com (Y. Yu), chen_wei74@163.com (W. Chen).

${ }^{1}$ These authors contributed equally to this work. 
Experimental factors Experimental features Data source location Data accessibility
Anticoagulant Peripheral blood with heparin Lithium IGRA-ELISA and ELISPOT tests

Xi'an, Shaanxi Province, Northwest China.

Data in the article can be accessible through LIS and HIS of the hospital

\section{Value of the data}

- This work shows the excellent consistency between T-SPOT.TB and IGRA-ELISA for diagnosing Mycobacterium tuberculosis infection.

- Data in this work demonstrates both T-SPOT.TB test and IGRA-ELISA can be used to rule out active TB.

- Data in this work indicates that the value change trend of the IGRA-ELISA was consistent with that of T-SPOT.TB test for the positive samples.

\section{Data}

\subsection{The performance of the two tests for diagnostic active $T B$}

The sensitivity and specificity for diagnostic active TB using T-SPOT.TB test were $82.9 \%$ and $78.6 \%$, and those by IGRA-ELISA were $81.7 \%$ and $75.2 \%$, Table 1 . The median comparison showed that the median value in active $T B$ (ATB) group was significantly higher than that in with nonactive TB (NATB) group for the two IGRAs tests; however, neither of the assays can be applied to diagnose active TB as they cannot differentiate active TB from NATB; see Fig. 1.

\subsection{Analysis of positive test results}

The positive results were divided into four groups according with the test values, and except for group 3, the proportion of positives was similarly distributed between the two tests (Table 2). The PPVs of the two tests at different cutoffs for diagnostic ATB were analyzed (Fig. 2).

\subsection{The consistency of the two tests}

204 patients were tested using both the T-SPOT.TB and IGRA-ELISA. The positive, negative, and total coincidence rates between the two tests were 95.5\% (84 of 88), 90.5\% (105 of 116), and 92.7\% (189 of 204 ), respectively.There was excellent consistency between the two tests with a kappa value of 0.852 $\left(\chi^{2}=148.64, p<0.0001\right)$. When 84 samples that were positive to both of the two tests were analyzed, we found that the value change trend of the IGRA-ELISA was consistent with that of T-SPOT.TB test, and the two curves intersected at approximately $50 \mathrm{pg} / \mathrm{mL}$ (IGRA-ELISA) and 30 SFCs (T-SPOT.TB).

\section{Table 1}

The performance of T-SPOT.TB and IGRA-ELISA for diagnosis ATB.

\begin{tabular}{|c|c|c|c|}
\hline \multirow[t]{2}{*}{ Performance } & \multicolumn{2}{|l|}{ Assays } & \multirow[t]{2}{*}{$P$ value } \\
\hline & T-SPOT.TB & IGRA-ELISA & \\
\hline Sensitivity:\% & 82.9 & 81.7 & 0.867 \\
\hline Specificity:\% & 78.6 & 75.2 & $0.023^{*}$ \\
\hline
\end{tabular}

\footnotetext{
" Statistically significant association, $P<0.05$.
} 
A

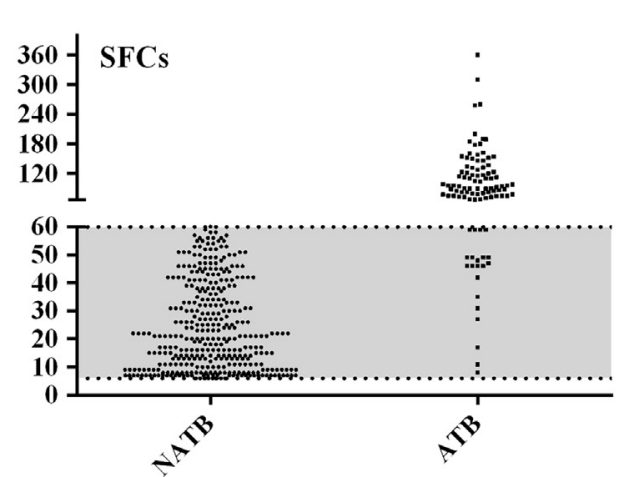

B

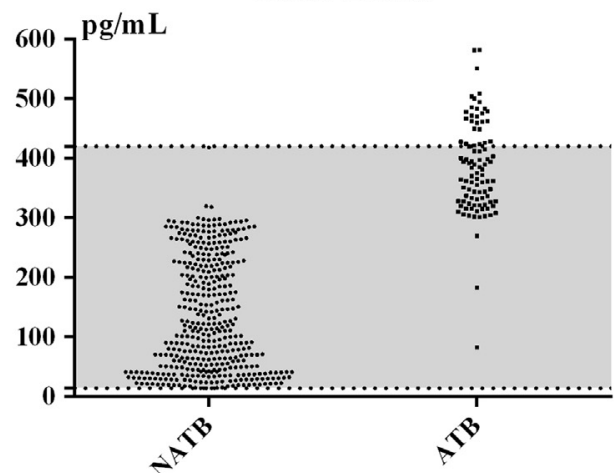

Fig. 1. The comparison of values between ATB group and NATB group in positive results for (A) T-SPOT.TB test and (B) IGRAELISA test. ATB was active tuberculosis; and NATB was nonactive tuberculosis.

Table 2

The analysis for positive results of T-SPOT.TB and IGRA-ELISA tests.

\begin{tabular}{|c|c|c|c|c|c|}
\hline \multirow[t]{2}{*}{ Groups } & \multicolumn{2}{|c|}{ IGRA-ELISA } & \multicolumn{2}{|l|}{ T-SPOT.TB } & \multirow[t]{2}{*}{$P$ value } \\
\hline & $\begin{array}{l}\text { Range: } \\
\mathrm{pg} / \mathrm{mL}\end{array}$ & Proportions(\%) & Range: SFCs & Proportions(\%) & \\
\hline Group 1 & $14-100$ & 38.06 & $6-20$ & 39.1 & 0.232 \\
\hline Group 2 & $100-200$ & 18.62 & $20-40$ & 22.46 & 0.689 \\
\hline Group 3 & $200-300$ & 24.29 & $40-60$ & 19.87 & $0.008^{*}$ \\
\hline Group 4 & $>300$ & 19.03 & $>60$ & 18.57 & 0.282 \\
\hline
\end{tabular}

* Statistically significant association, $P<0.05$.

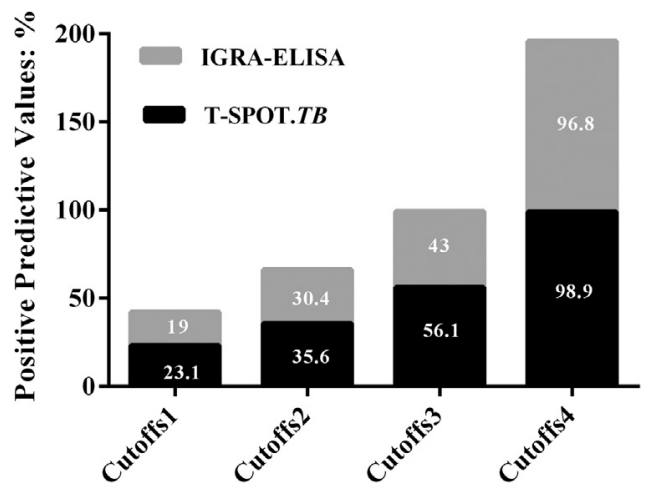

Fig. 2. The positive predictive value of T-SPOT.TB and IGRA-ELISA test at cutoff 1 , cutoff 2 , cutoff 3 and cutoff 4 . Cutoff $1,2,3$, 4 for T-SPOT.TB were 6, 20, 40 and 60 SFCs; those for IGRA-ELISA were 14, 100, 200 and $300 \mathrm{pg} / \mathrm{mL}$.

\section{Experimental design, materials and methods}

\subsection{Enrolled population}

A total of 3727 patients with suspected Mycobacterium tuberculosis infection at the First Affiliated Hospital of Xi'an Jiaotong University were enrolled. Among them, 204 were tested using both the T-SPOT.TB and IGRA-ELISA, 1794 were tested using the T-SPOT.TB only, and 1729 were tested using the 
IGRA-ELISA only. The median age was 55 years, the majority of participants ( $n=2100$ [56.3\%]) were men. 244 patients were diagnosed with active TB if at least one of the following conditions were fulfilled: (i) smear-positive for acid-fast bacilli or TB PCR-positive, $n=19$, (ii) chest X-ray findings consistent with a radiological diagnosis of PTB, $n=53$, (iii) granulomata or caseous necrosis observed on histopathological examination of biopsy specimens, $n=45$, or (iv) despite the absence of explicit evidence of active TB on microscopy, TB PCR, chest X-ray, or histopathology, the clinical symptoms were consistent with active TB and responded to anti-TB treatment $(n=127) .129$ and 115 of the patients underwent the T-SPOT.TB and IGRA-ELISA testing, respectively.

\subsection{Performing the T-SPOT.TB and IGRA-ELISA test}

Peripheral blood samples from the participants were collected in lithium-heparin-coated tubes. The T-SPOT.TB test (Oxford Immunotec Ltd., Abingdon, UK) was performed within $6 \mathrm{~h}$ of obtaining the blood sample. The separation and incubation of peripheral blood mononuclear cells were conducted as previously reported [1]. The IGRA-ELISA (WanTai Biological Pharmacy Enterprise Co, Ltd., Beijing, China) was performed within $2 \mathrm{~h}$ of obtaining the blood sample. $1 \mathrm{~mL}$ of blood was added to each of 3 heparinized tubes (T, N, and P tube). The tubes were incubated at $37^{\circ} \mathrm{C}$ for $20-24 \mathrm{~h}$ and were then centrifuged at 3000-5000 rpm for $10 \mathrm{~min}$. The supernatant was used for the ELISA assay, performed using an automated instrument.

\section{Acknowledgments}

None.

\section{Transparency document. Supporting information}

Transparency data associated with this article can be found in the online version at https://doi.org/ 10.1016/j.dib.2018.08.112.

\section{Appendix A. Supporting information}

Supplementary data associated with this article can be found in the online version at https://doi. org/10.1016/j.dib.2018.08.112.

\section{Reference}

[1] Linchuan Wang, Yan Yu, Wei Chen, Jin Feng, Jinyuan Wang, He Ping Zhao, Lietin Ma, Bo Yang, Yanfen Ma, Pei Dang, Evaluation of the characteristics of the enzyme-linked immunospot assay for diagnosis of active tuberculosis in China, Clin. Vaccine Immunol. 22 (5) (2015) 510-515. 